\title{
Propuesta de automatización para el seguimiento de ventas en microempresas
}

Fecha de recepción: 2021-07-31 • Fecha de aceptación: 2021-09-10 • Fecha de publicación: 2021-10-10

\author{
Juan Sebastián Reza Morales \\ Tata Consultancy Services, Ecuador \\ jsrezam@gmail.com \\ https://orcid.org/0000-0002-8318-3695
}

\section{RESUMEN}

Varias microempresas realizan gran parte de la comercialización de sus productos de forma manual, sin una gestión de información óptima. En muchas ocasiones, el almacenamiento de estos datos es nulo. Registrar el trabajo realizado a lo largo de los principales flujos y acciones que se dan al momento que el agente de ventas contacta al cliente, oferta el producto y registra una orden de compra, es clave a razón de generar una visión concreta e integra acerca del desempeño real de los colaboradores, sin dejar de lado que pone a disposición una estadística global del negocio. La automatización de los procesos que realizan los agentes para llevar a cabo el registro de órdenes incrementará la eficiencia y la productividad de este flujo. Toda esta información automatizada, gestionada y almacenada a través de un sistema, brindará a la gerencia facilidad en la toma de decisiones que potenciarán el auge del negocio.

\section{PALABRAS CLAVE: automatización, sistema web, productividad.}

\section{ABSTRACT}

Several micro-companies carry out much of the marketing of their products manually without optimal information management. In many cases, the storage of this data is null. Registering the work carried 
out along the main flows and actions that occur when the sales agent contacts the customer, offers the product, and registering a purchase order is key to generate a specific and integrated vision about performance real of the collaborators, without neglecting that it makes available a global statistics of the business. Automating the processes that agents carry out to carry out an order registration will increase the efficiency and productivity of this flow. All this automated information, managed and stored through a system, will provide management power in making decisions that will boost the business.

KEYWORDS: automation, web system, productivity. 


\section{Introducción}

En un entorno cada vez más competitivo, es inaceptable realizar procesos manuales, el depender de un almacenamiento de información volátil, poco confiable y en muchos de los casos, hasta indisponible, impide a las gerencias o mandos superiores del negocio conocer el estatus real del mismo (Trasobares, 2003). Este problema puede llegar a limitar el crecimiento u optimización de las actividades primordiales del flujo de ventas.

Actualmente existen pequeños negocios dedicados a la venta y comercialización de sus respectivos productos, y para automatizar sus procesos utilizan sistemas informáticos, en su gran mayoría, sistemas web (Riera, 2020). Esta actividad regularmente es realizada por los agentes vendedores, los cuales se desenvuelven bajo la supervisión de un agente senior, cuya función consiste en recopilar toda la información relacionada con el desempeño de sus supervisados a lo largo de la interacción con el flujo de ventas. La constante implementación de soluciones tecnológicas tiende a dejar atrás la sensación de ineficiencia, que se podría dar al momento de ejecutar las actividades necesarias para ofertar y vender los productos relacionados al negocio.

La literatura demuestra que la automatización mediante sistemas informáticos se ha explorado notablemente, así lo demuestran varios trabajos publicados. Inicialmente se puede mencionar a Trasobares (2003), que desarrolla un sistema para gestión de ventas en unidades móviles, permitiendo a la agencia de venta ofrecer una fuente de información a través de la web, actualmente por la pandemia COVID-19, la mayoría de empresas optaron por utilizar sistemas móviles para optimizar sus procesos. Reyes Rivera et al. (2020) mencionan la problemática de las pequeñas empresas en tiempos de pandemia y proponen una solución informática para que la empresa pueda realizar sus ventas en línea, Artieda Guachamin et al. (2021) proponen una aplicación móvil personalizada para ofertar transporte en la ciudad de quito con características similares Uber e Indriver, en aplicaciones móviles se puede mencionar varias soluciones para gestionar adopciones de mascotas callejeras, que en los últimos años en la ciudad de quito son un gran problema para la sociedad (Burbano Ulloa et al., 2020).

En este sentido, el presente trabajo ha sido construido en base a una sólida especificación de requerimientos indispensables para resolver un problema que se da cotidianamente en las empresas dedicadas a la oferta y venta de productos. El desarrollo realizado para este trabajo se enfocó directamente a pequeños negocios, los cuales buscan una automatización económicamente accesible y acorde a su volumen de productos, clientes y generación de órdenes para flujos como: control y seguimiento de campañas de venta, registro de órdenes y contactabilidad al cliente. El seguir una arquitectura enfocada en el ágil desacoplamiento del sistema implica una mayor inversión de tiempo al momento de generar nueva funcionalidad, debido al mandatorio construcción de interfaces necesarias para establecer comunicación con las diferentes capas del software.

En contraste al aplicar este tipo de arquitectura, se genera también una mejor mantenibilidad en el software, y lo más importante, la deuda técnica del sistema disminuye considerablemente, ya que al seguir un mismo estándar de desarrollo especializado en conservar la independencia 
de componentes, resulta en impactos leves para mantenimientos futuros, como, por ejemplo, el cambio de la herramienta ORM por cambio de tecnología front-end.

\section{Metodología}

El proyecto se ejecutó bajo la metodología ágil Scrum, a razón de aprovechar que la misma involucra al cliente y ayuda a generar entregas parciales del desarrollo en un periodo de tiempo predeterminado llamado sprint.

Para la ejecución de la metodología Scrum se utilizó la herramienta “Azure Devops”, con el objetivo de aprovechar los servicios que ofrece para los equipos de soporte y desarrollo. Con esta herramienta se puede planificar el trabajo, generar colaboración en el desarrollo de código y crear e implementar aplicaciones (Microsoft, 2021).

En específico de todos los servicios que presenta, está Suit, se empleó la funcionalidad del servicio "Azure Boards", el cual resulta beneficioso para generar la administración del proyecto, ya que maneja un soporte nativo para la ejecución de la metodología Scrum, sin dejar de lado que se apoya en el tradicional tablero Kanban útil para generar el respectivo seguimiento a las tareas de cada iteración.

En la siguiente Tabla 1 se visualizará la lista de requerimientos presentados para este proyecto.

\section{Tabla 1}

Tabla de Requerimientos

\begin{tabular}{|l|l|}
\hline & \multicolumn{1}{|c|}{ Tabla de Requerimientos } \\
\cline { 2 - 3 } & \multicolumn{1}{|c|}{ Requerimientos } \\
\hline 1 & $\begin{array}{l}\text { El sistema debe contar un módulo que gestione la autenticación y autorización de los usuarios en el sistema. } \\
\text { existentes en el sistema. }\end{array}$ \\
\hline 3 & $\begin{array}{l}\text { El sistema debe contar con un módulo que gestione la carga, consulta y modificación de los productos } \\
\text { existentes en el sistema. }\end{array}$ \\
\hline 4 & $\begin{array}{l}\text { El sistema debe contar con un módulo que gestione la carga, consulta y modificación de los clientes existentes } \\
\text { en el sistema. }\end{array}$ \\
\hline 5 & $\begin{array}{l}\text { El sistema debe contar con un módulo que gestione el estado y los privilegios de los usuarios existentes en el } \\
\text { sistema. }\end{array}$ \\
\hline 6 & $\begin{array}{l}\text { El sistema debe contar con un módulo que gestione la creación y consulta de las órdenes a transaccionarse en } \\
\text { el sistema. }\end{array}$ \\
\hline 7 & $\begin{array}{l}\text { El sistema debe contar con un módulo que gestione la creación, asignación, consulta y modificación de } \\
\text { campaña de ventas. }\end{array}$ \\
\hline 8 & $\begin{array}{l}\text { El sistema debe contar con un dashboard que muestre la siguiente información sobre la actividad de ventas } \\
\text { ejecutada: los productos más vendidos en el mes, los clientes más destacados en el mes, el agente más } \\
\text { destacado, un chart con la información de ventas realizadas por cada agente y finalmente un chart que } \\
\text { destace el total de ventas por mes. }\end{array}$ \\
\hline
\end{tabular}


$9 \quad$ El sistema debe contar una aplicación web en la cual el agente pueda gestionar la contactabilidad al cliente y el progreso en sus campañas activas.

A su vez, en la Tabla 2 se evidencian los roles e integrantes.

Tabla 2

Tabla de Scrum Team

\begin{tabular}{|c|c|c|}
\hline & \multicolumn{2}{|c|}{ Scrum Team } \\
\hline 1 & Sebastián Reza & $\begin{array}{c}\text { Scrum Master / Development Team } \\
\text { Member }\end{array}$ \\
\hline
\end{tabular}

La construcción del presente proyecto tomó de tres iteraciones para abarcar todas las características y funcionalidad

\section{Primer sprint}

El primer sprint fue ejecutado del 3 al 10 de mayo de 2021, cubriendo tareas iniciales indispensables a razón de establecer los fundamentos de la arquitectura limpia, la cual busca que el core del negocio que maneja la aplicación sea altamente desacoplable, tanto a los frameworks ORM, como a los frameworks que se especializan para construir front-end.

Otro hito importante para este sprint fue establecer las relaciones entre objetos de negocio para la gestión de usuarios, sin dejar de lado las validaciones de autenticación y autorización al sistema. A continuación, en la Figura 1 se muestra parte del backlog ejecutado.

\section{Figura 1}

Backlog para el primer sprint en Azure DevOps

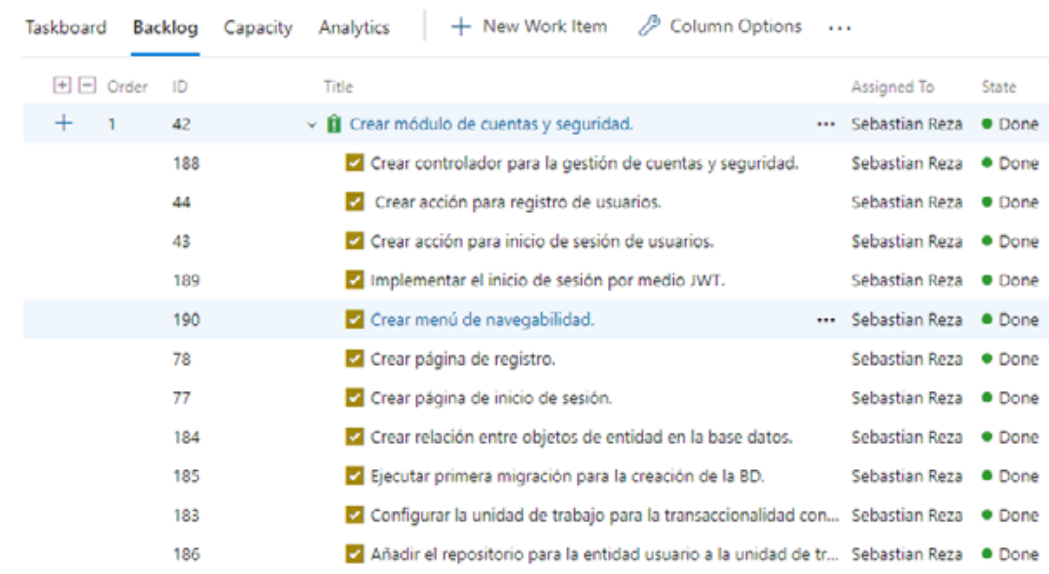




\section{Segundo sprint}

El segundo sprint (Figura 2) fue ejecutado del 11 al 27 de mayo de 2021, con el objetivo de implementar la funcionalidad medular del sistema, la misma que se basa en habilitar al supervisor de ventas la posibilidad de generar campañas, las cuales cuenten con un número finito de clientes afines al negocio, para posteriormente ser contactados por un agente de ventas, a quien será asignada la nueva campaña creada por el supervisor.

Otros entregables importantes de esta iteración fueron la construcción de los módulos que gestionan los objetos de negocio como categorías, productos y clientes.

Figura 2

Backlog para el segundo sprint en Azure DevOps

\begin{tabular}{|c|c|c|c|c|c|c|c|}
\hline Taskboard & & klog & Copacity & + New Work Item & Column Options & & \\
\hline$\boxplus \boxminus$ & Order & 10 & & Title & & Assigned To & State \\
\hline+ & 1 & 47 & & , î Crear módulo de campañas. & $\cdots$ & Sebastian Reza & - Done \\
\hline & 2 & 49 & & > î Crear módulo de clientes. & & Sebastian Reza & - Done \\
\hline & 3 & 46 & & D (î Crear módulo de categorías. & & Sebastian Reza & - Done \\
\hline & 4 & 45 & & > î Crear módulo de productos. & & Sebastian Reza & - Done \\
\hline
\end{tabular}

\section{Tercer sprint}

El tercer sprint (Figura 3) fue ejecutado del 28 de mayo al 30 de junio del 2021, llevando a cabo la construcción de la aplicación móvil, la misma que será utilizada como herramienta por el agente de ventas para desarrollar sus campañas asignadas y facilitar la contactabilidad a los clientes presentes, en su respectivo detalle de ofertas. Finalmente, la implementación de un tablero de reportes, el cual muestra información relevante acerca de la ejecución del flujo de ventas, añadiéndole la construcción del módulo que gestiona la creación y consulta de órdenes, tanto a nivel de agente, como de administrador.

\section{Figura 3}

Backlog para el tercer sprint en Azure DevOps

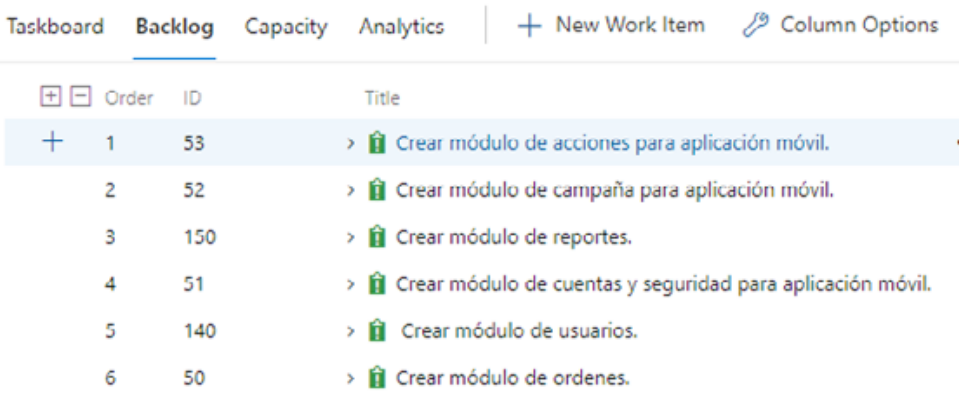

\footnotetext{
Assigned To State

... Sebastian Reza - Done

Sebastian Reza • Done

Sebastian Reza - Done

Sebastian Reza - Done

Sebastian Reza - Done

Sebastian Reza - Done
} 
En la Figura 4 se muestra el modelo físico de la base de datos empleada por el sistema para realizar la persistencia de datos.

Figura 4

Base de datos del sistema

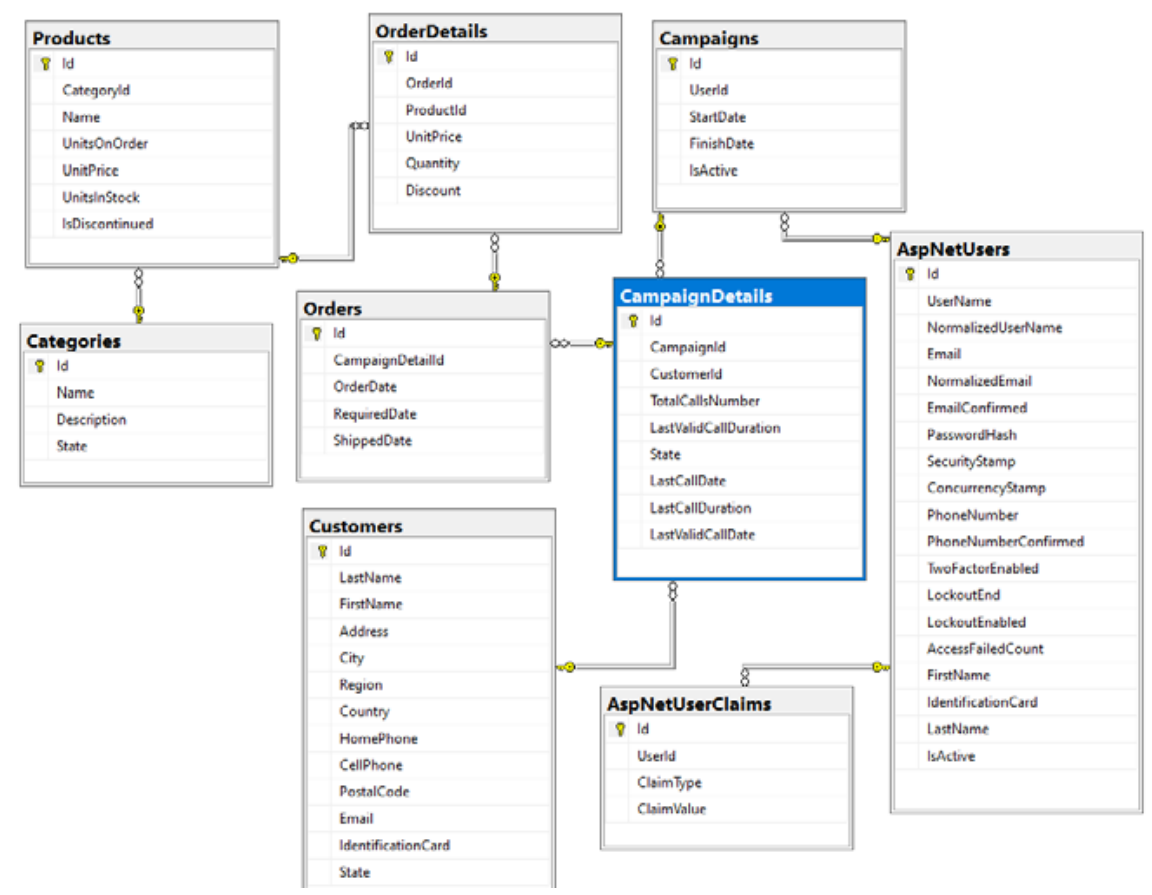

En la siguiente Figura 5 se expone la arquitectura que emplea el sistema a nivel de hardware y software utilizados. 
Figura 5

Infraestructura de la solución

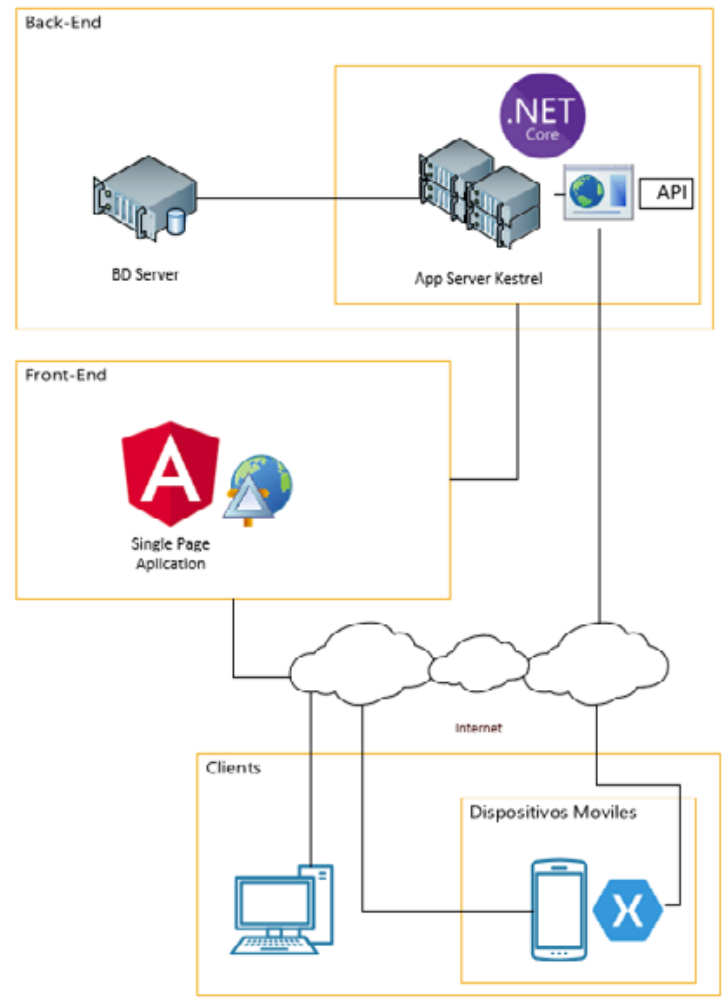

A continuación, se presenta la arquitectura en la que se desenvuelve la transaccionalidad del sistema, básicamente se compone de los siguientes elementos de hardware y software:

- Back-end: es la parte de la solución que se conforma por todo el hardware y software encargado de gestionar la información que ingresa por las diferentes interfaces que expone el sistema. El back-end posibilita el acceso a las operaciones de configuración del sistema y de las funcionalidades puestas a disposición (Solon et al., 2015).

- Servidor de base de datos: es la infraestructura en la cual se apoya el motor de base de datos relacional, el cual se encarga de gestionar y persistir las transacciones que demande el sistema. Para este desarrollo se ha seleccionado SQL Server 2019 como motor de base de datos, estos componentes conforman la parte del back-end de la solución.

- Servidor de aplicaciones: es la infraestructura en la cual se apoya el API RESTful, el mismo que se encarga de exponer toda la funcionalidad que las interfaces de la aplicación consumen; con el objetivo de consultar, transaccionar y mostrar toda la información que se genera en los flujos del sistema, para la construcción de este componente su utilizo la tecnología .Net Core (Paco et al., 2009).

- API: es un acrónimo del término inglés Application Programming Interface (en español: interfaz de programación de aplicaciones). Su principal característica es que permiten que 
sus productos se comuniquen con otros sin que estos conozcan su implementación. Su funcionamiento se considera que es parecido a un contrato, ya que, si el emisor envía una solicitud con una estructura conocida, el receptor podrá realizar la operación pertinente de forma adecuada y devolver una respuesta con un cierto formato establecido (de la Maza Valles, 20209.

- .Net Core: es un marco multiplataforma de alto rendimiento y código abierto para crear aplicaciones modernas, habilitadas para la nube y conectadas a Internet (Microsoft, 2021).

- Front-end: es el componente del sistema que se encuentra disponible al público (Solon et al., 2015), encargado de receptar y enviar al back-end todas las transacciones que demanda el usuario y sin dejar de lado que es el mismo componente que se encarga de mostrar la información solicitada el mismo.

- Single page application: aplicación web que carga completamente todos los recursos en la solicitud inicial y luego los componentes de la página son reemplazados por otro componente dependiendo de la interacción del usuario es decir no se da un recargo de toda la página para cada petición (Jadhav et al., 2015).

- Angular: es una plataforma de desarrollo construida sobre TypeScript, con un marco basado en componentes para crear aplicaciones web escalables, cuenta con una colección de bibliotecas bien integradas que cubren una amplia variedad de características, que incluyen enrutamiento, administración de formularios, comunicación cliente-servidor entre algunas otras (Angular, s.f).

- TypeScript: es un lenguaje de programación basado totalmente en JavaScript que se enfoca en producir código seguro y predecible y este puede ser ejecutado por cualquier tiempo de ejecución de JavaScript. Su característica principal es la escritura estática, que hace que trabajar con JavaScript sea más predecible y cómodo para los programadores familiarizados con lenguajes tipados (Freeman, 2021).

- Xamarin: plataforma de código abierto para crear aplicaciones modernas y eficaces para iOS, Android y Windows con .NET. Xamarin es una capa de abstracción que administra la comunicación del código compartido con el código de la plataforma subyacente. Este patrón permite a los desarrolladores escribir toda su lógica empresarial en un solo idioma (o reutilizar el código de la aplicación existente) pero lograr un rendimiento, una apariencia y un funcionamiento nativos en cada plataforma (Microsoft, s.f).

- Clean Arquitecture: esta arquitectura se empleó específicamente para la construcción del API Rest, lo que busca es que el core del negocio que maneja la aplicación sea altamente desacoplable tanto a los frameworks ORM, como a los frameworks que se especializan para construir front-end. Uno de los beneficios importantes que podemos destacar de esta arquitectura es que al no tener lógica de negocio en la base de datos se puede tratar a este componente como un simple repositorio, al cual el núcleo del negocio se puede desvincular cuando la organización lo crea conveniente, de la misma forma funcionará el desacoplamiento al framework encargado de gestionar el front-end, porque de igual manera, la arquitectura direcciona a la inexistencia de lógica de especializada para el núcleo del negocio en la capa de presentación. 
Aplicando clean architecture y respetando las normas establecidas, independientemente de si posteriormente se implementa mejor o peor, como mínimo obtendremos un software robusto, el cual nos proporcionará un software fácil de mantener, puesto que el desacoplamiento que proponen las capas brindará a nuestro programa del principio SOLID Open/Closed, lo que quiere decir que estará abierto a extensión, pero cerrado a modificación; conseguiremos que el software sea propio y no dependa de cambios externos (González, 2016).

\section{Resultados}

El sistema se basó en dos perfiles de usuario indispensables para poder esquematizar, organizar y persistir almacenar la información del flujo de ventas. La siguiente Tabla 3 muestra las actividades que desempeña cada perfil en el sistema.

\section{Tabla 3}

Tabla de Perfiles de usuario

\begin{tabular}{|l|l|l|l|}
\hline Nombre de Usuario & Tipo de Usuario & Área Funcional & \multicolumn{1}{|c|}{ Actividad } \\
\hline Administrador & Administrador & Administración & Administrar clientes. \\
& & & Administrar categorías. \\
& & & Administrar productos. \\
& & Administrar usuarios. \\
& & Administrar campañas. \\
& & Visualizar progreso campañas. \\
& & Visualizar dashboard. \\
& & Visualizar órdenes. \\
& & Administrar clientes. \\
& Agente de Ventas & Ventas & Gestionar campañas asignadas. \\
\hline Agente & & & Gestionar campañas asignadas. \\
& & & Sincronizar llamadas del día. \\
& & & Generar órdenes. \\
& & & Visualizar solamente sus órdenes \\
& &
\end{tabular}

Con el fin de agilizar el proceso de asignación de clientes, que tiene por objetivo realizar la oferta de productos o promociones del negocio, el sistema propone el siguiente flujo que se muestra en la Figura 6: 


\section{Figura 6}

Casos de uso para la creación, asignación y consulta de campañas

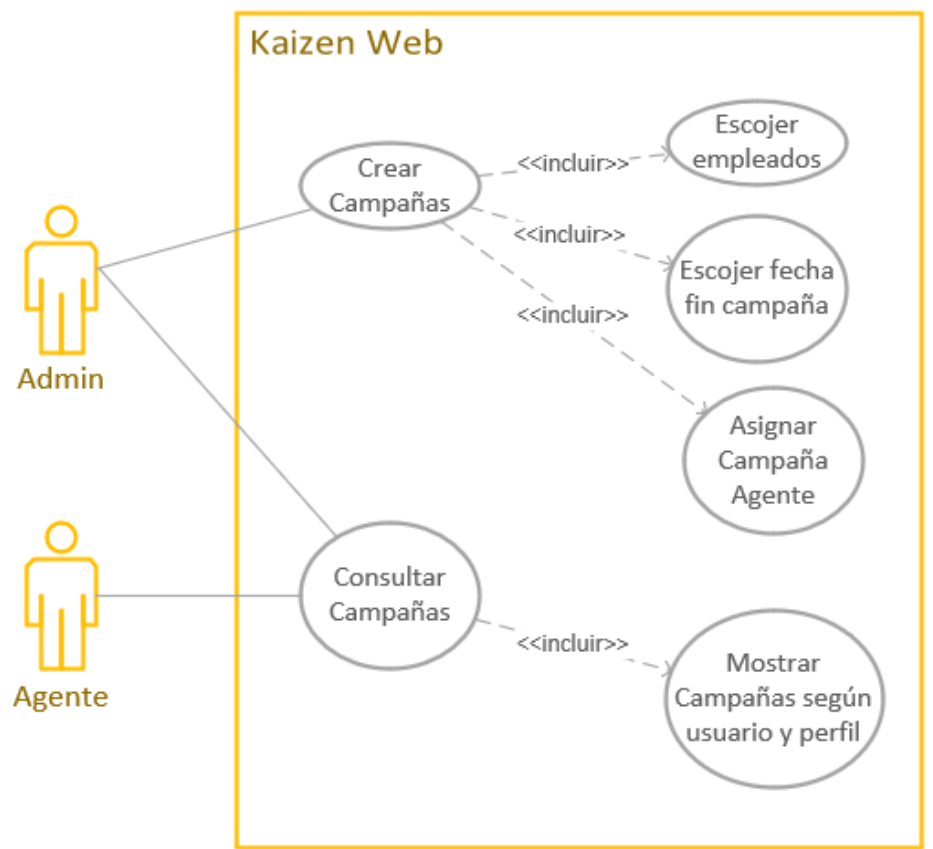

El usuario administrador ingresa al sistema para ejecutar la acción que lleva a cabo la creación de una nueva campaña. Esta acción debe cumplir con tres pasos subsecuentes los cuales son: elegir los empleados que conformarán la campaña, seleccionar una fecha fin para la campaña y escoger un agente a quien otorgarle la nueva campaña a crearse.

Con esta implementación se evita el manejo de archivos tipo hojas de cálculo, archivos planos, e incluso, libros de registro que estén destinados a manejar esta información de forma deficiente. De esta forma, el sistema es el único gestor de la información que fluye para los objetos principales de esta funcionalidad, como lo son las campañas y los clientes.

Otro punto importante que se gana con esta funcionalidad en el sistema es que se evita perdidas de información, ya sea en una transmisión por correo electrónico o físicamente, referente a estas asignaciones de trabajo, ya que, de manera automática e inmediata, la campaña que asigna el administrador por medio del sistema aparecerá en la bandeja del usuario agente para que sea trabajada.

Es importante recalcar que el administrador puede consultar y ver el progreso de cualquier campaña perteneciente a cualquier agente en el ámbito del sistema, lo cual resulta sumamente beneficioso para que el administrador cuente con un conocimiento real del avance de sus colaboradores. 
Las siguientes Figuras 7 y 8 muestran el aspecto del formulario para crear y asignar campañas del lado del usuario administrador, como de la página que muestra la bandeja que expone estas campañas asignadas del lado del usuario agente.

\section{Figura 7}

Página creación y asignación de campañas perfil administrador

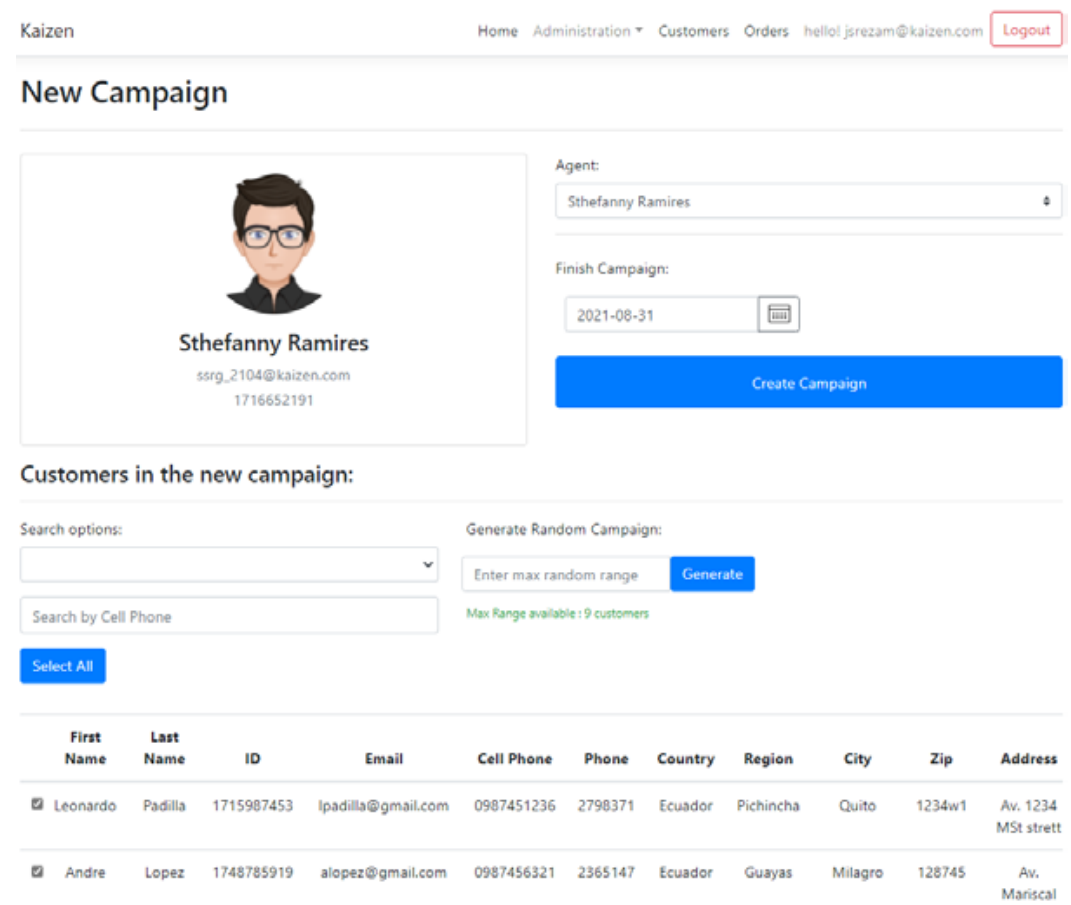

\section{Figura 8}

Página bandeja de campañas asignadas perfil agente

Kaizen

My campaigns

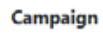

1100
Start Date

$8 / 24 / 21,11: 56$ PM
Home Customers My Campaigns Orders

logout 
El perfil agente podrá ver el detalle de su campaña a desarrollar en la página de desglose de campaña (Figura 9), la misma que mostrará para cada oportunidad presente en la campaña los clientes a contactar.

\section{Figura 9}

Página desglose de campaña perfil agente

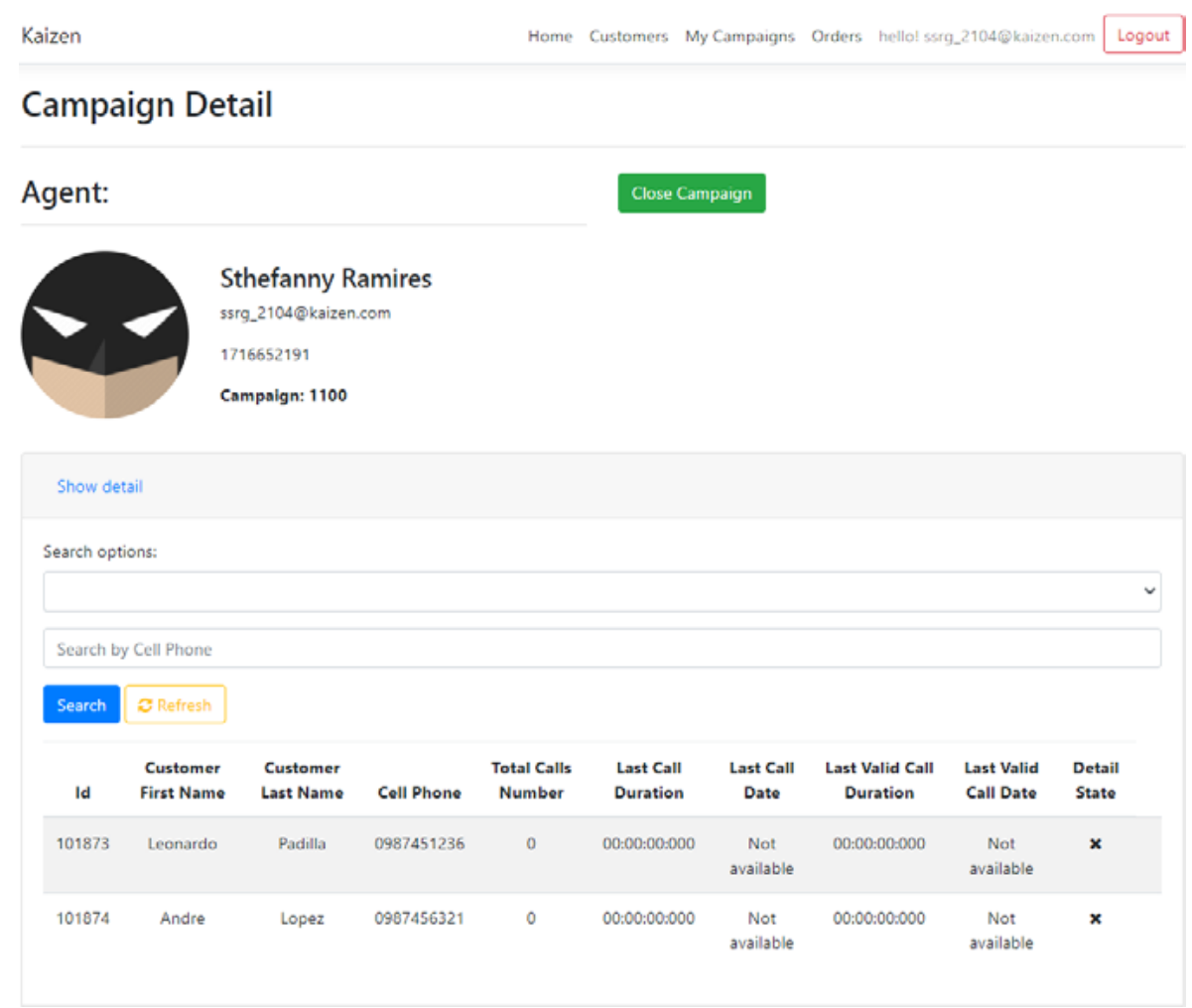

Existen los siguientes cuatro estados (Figura 10) que pueden tomar las oportunidades según las circunstancias que se exponen a continuación:

- Uncalled: la oportunidad tiene este estado cuando el agente aún no se ha contactado con el cliente presente en la oferta.

- Called: la oportunidad tiene este estado cuando el cliente ya se contactó con el cliente presente en la oferta.

- Losted: la oportunidad tiene este estado cuando la campaña se cerró y la oferta no concretó una generación de orden.

- Earned: la oportunidad tiene este estado cuando la oferta se concretó con una generación de orden. 
Figura 10

Estados de la oferta
X Uncalled
(")) Called
$\$$ Losted
Garned

Otra funcionalidad que aporta significativamente al negocio es cuando el agente crea o concreta pedidos (Figura 11). Con la correcta estructuración y gestión de la información que propone el sistema, este brinda agilidad, seguridad, integridad y disponibilidad a estas transacciones, lo cual mejora significativamente a comparación del trato manual con estas acciones, ya que, al manejar archivos sueltos sin una debida seguridad, ni un confiable almacenamiento, existe un riego bastante grande de perdida de información o modificación no autorizada de datos.

\section{Figura 11}

Casos de uso para generar órdenes

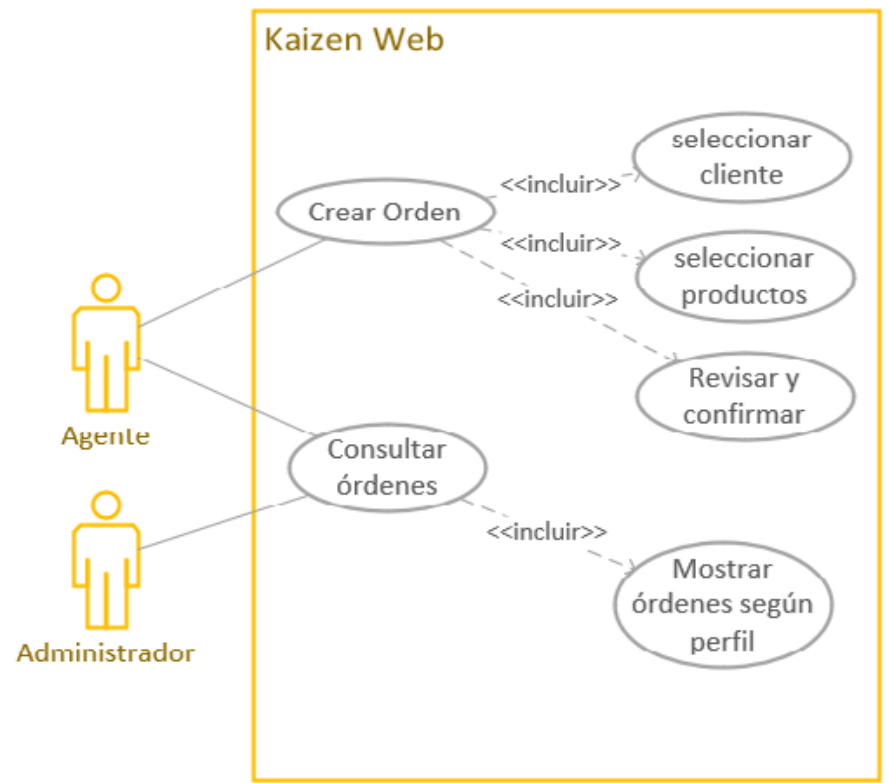

Para crear una orden el agente tendrá que escoger un cliente que exista en su campaña en progreso, asignarle los productos en los que esté interesado, y como paso final, validar el detalle 
generado con sus respectivos montos, cantidades e información básica del cliente en una interfaz de check-out.

Como se puede observar en el caso de uso el usuario administrador, este tendrá acceso read-only a cualquier orden generada por cualquier agente existente en el ámbito del sistema, a diferencia del usuario agente de ventas que solamente podrá consultar sus propias ordenes generadas.

Las siguientes Figuras 12, 13 y 14 muestran el aspecto de las interfaces para generar una orden en sus tres etapas: selección de cliente, selección de productos y check-out de orden.

\section{Figura 12}

Página para generar órdenes interfaz de selección de cliente

Kaizen

Home Customers My Campaigns Orders hello: ssrg__2104థkaizen.com Logout

New Order
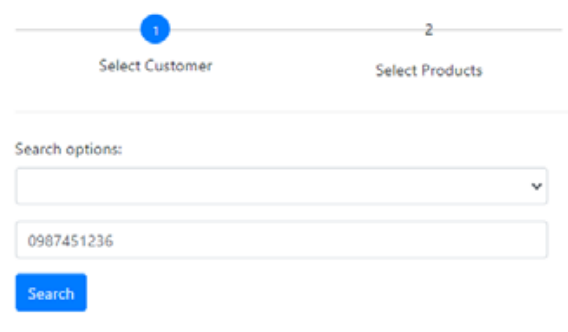

Selected Customer:

\section{Search}

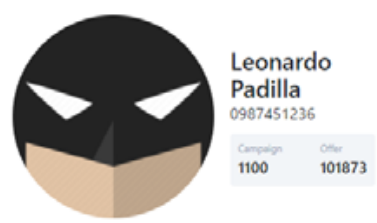




\section{Figura 13}

Página para generar órdenes interfaz de selección de producto

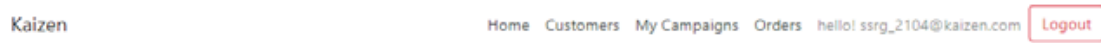

New Order

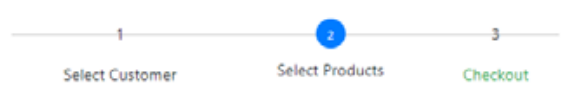

Search options:

Cart Resume:

\begin{tabular}{|llr}
\hline & teems: & ( \\
\hline Lenovo Chromebook S330 portatil & Total (USD): & 5773.97
\end{tabular}

Search

\begin{tabular}{|ccccccc|}
\hline Product ld & Category & Name & Unit Price & Stock & Units on Order \\
\hline 82875 & Technology & Lenovo Chromebook S330 portstil & 5257.99 & 75 & 24 & + \\
\hline
\end{tabular}

Items:

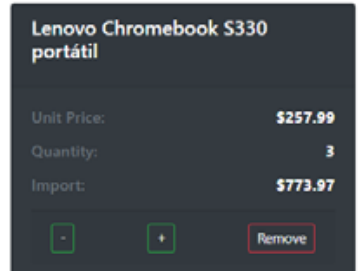

Figura 14

Página para generar órdenes interfaz de check-out

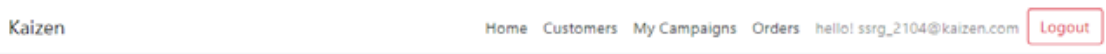

New Order

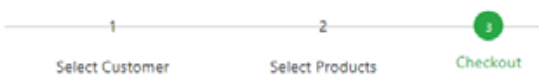

Customer Info:

Customer's Cart

1

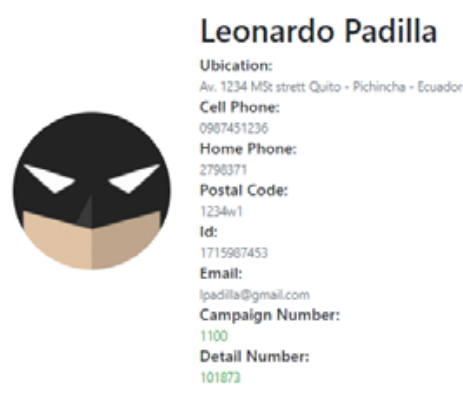

Lenovo Chromebook \$330 portátil $\quad \$ 773.97$ $525799 \times 3$
Total (USD) $\quad \$ 773.97$ 
Finalizando con la parte web del sistema, las siguientes imágenes muestran el tablero informativo (Figura 15) sobre las ventas efectuadas en el flujo. Esta interfaz da a conocer varios puntos importantes para definir el estatus actual del negocio.

Figura 15

Página que muestra el dashboard informativo
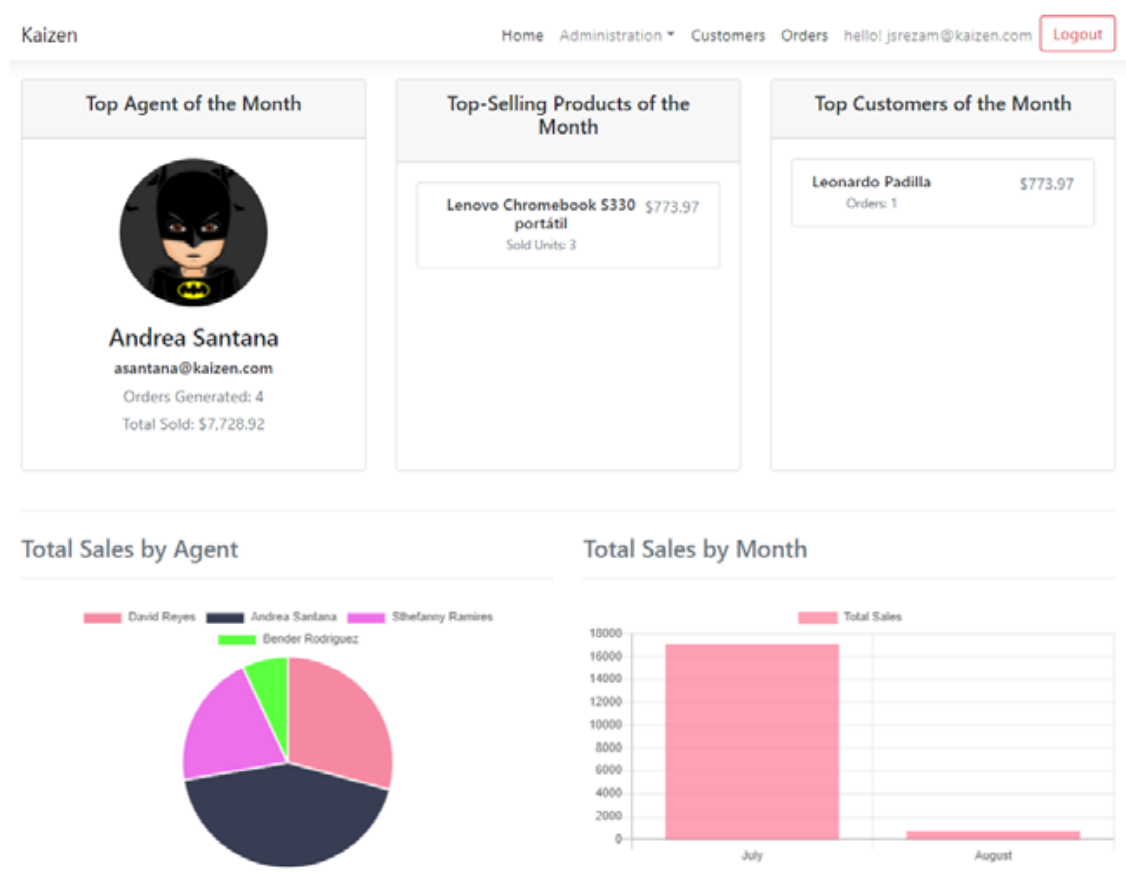

Total Sales by Month

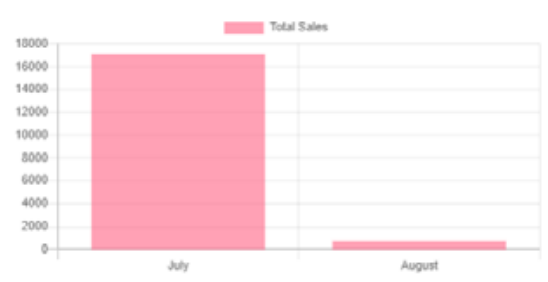

Con el fin de generar veracidad al momento de contactar con los diferentes clientes, se presentan las oportunidades de la campaña, se ha desarrollado un aplicativo móvil, el cual tiene por objetivo sincronizar la información que se genera en el dispositivo móvil al momento de realizar llamadas (Figura 16), con esta funcionalidad, el administrador podrá saber cuántas veces intento el agente contactarse con el cliente y cuánto duro su última llamada. 


\section{Figura 16}

Casos de uso para generar la sincronización de la información de llamadas

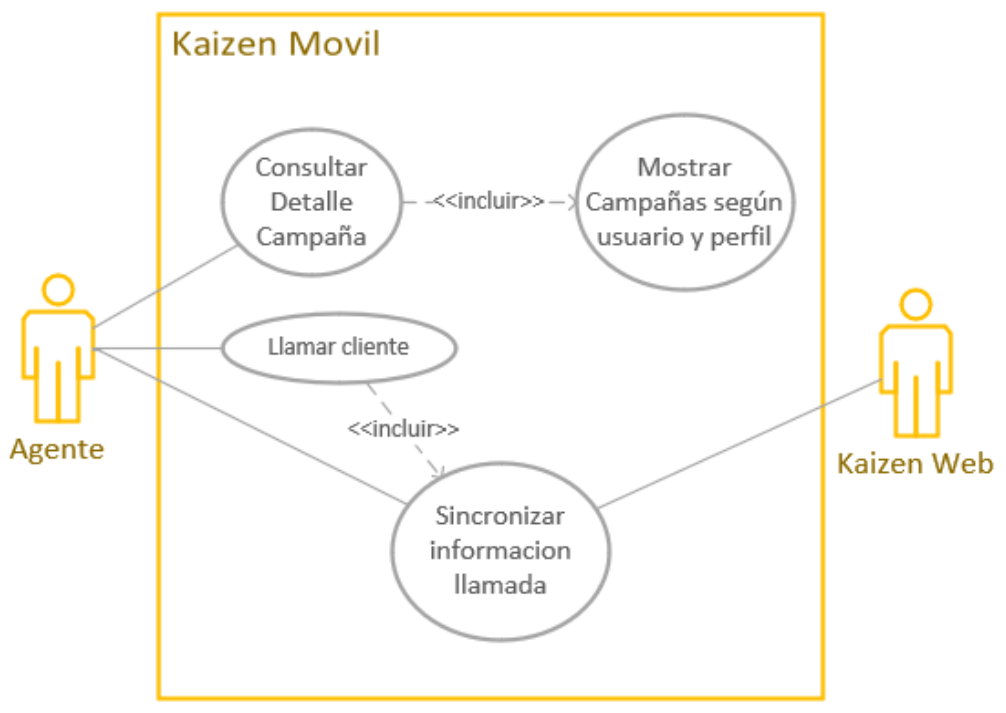

Como se puede observar en el caso de uso, el aplicativo web, al utilizar el mismo API que gestiona la información en el mismo repositorio de datos, también quedará actualizado con la información de las llamadas realizadas por el agente, al momento de ejecutar estas acciones de sincronización desde la aplicación del dispositivo móvil. Las siguientes Figuras 17 y 18 muestran el aspecto de las interfaces del aplicativo móvil, al momento que se realiza una sincronización de llamadas. 


\section{Figura 17}

Pantallas que muestran la campaña con su respectivo desglose

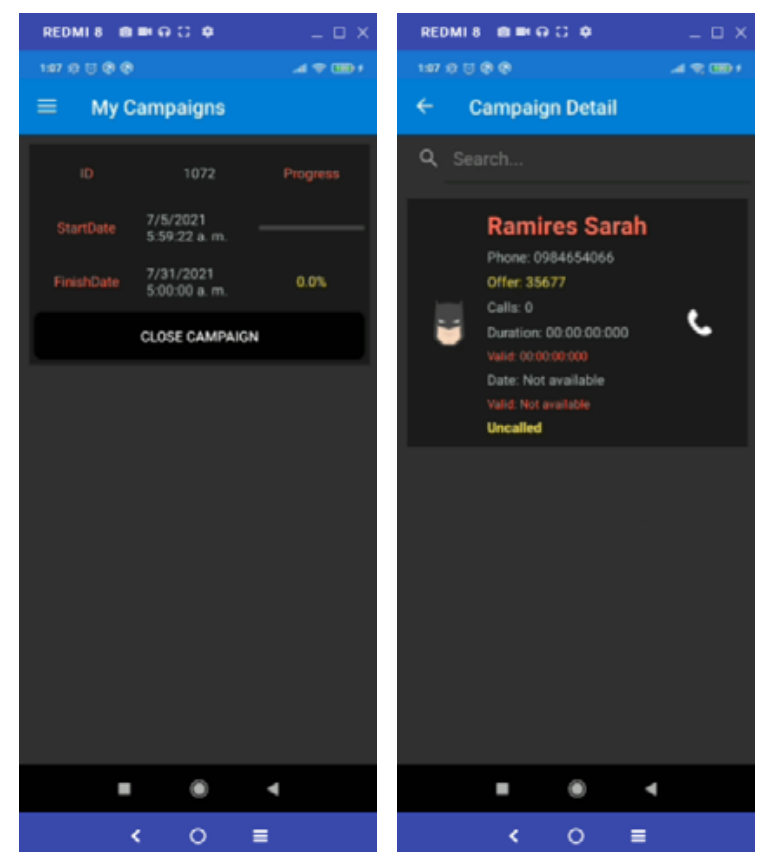

Figura 18

Sincronización de información de llamadas
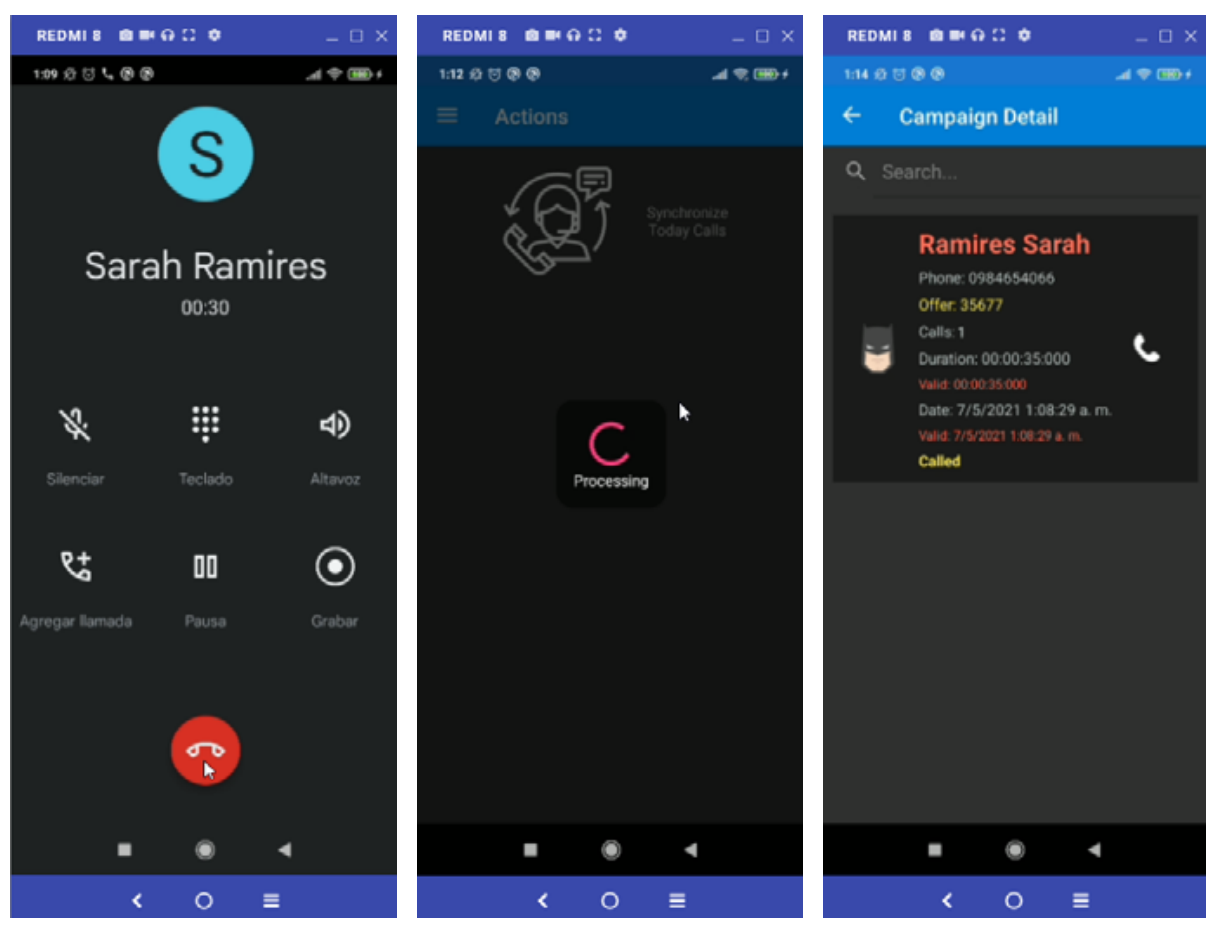
Consumando el resultado positivo para cada uno de los siguiente puntos clave que busca cubrir el sistema:

- Reducción de trabajo manual.

- Organización y estructuración correcta de la información que genera el negocio.

- Asegurar la integridad en la información que genera el negocio.

- Brindar seguridad a la información y funciones que resguarda el sistema.

\section{Conclusiones}

Sin duda, el sistema construido aliviana un alto porcentaje el trabajo manual que existe en el ámbito de ventas. También se enfoca en remover las inconsistencias que produce un flujo operado sin una debida seguridad administrable ni una gestión de persistencia apropiada de los datos. Este software, en su operación diaria, generará información valiosa y confiable, que, a su tiempo, ayudará a las gerencias de estos pequeños negocios con la toma de decisiones que los enruten al siguiente paso tecnológico.

Respaldar y solidificar la funcionalidad de la aplicación es uno de los puntos medulares de cualquier proyecto de software. Es de imperiosa necesidad asegurar que el producto este probado bajo un contexto correcto de carga y estrés transaccional, con el objetivo de evitar lentitud, y en el peor de los casos, la indisponibilidad del sistema en un ambiente productivo. 


\section{Referencias}

Angular. (s. f.). What is Angular? https://angular.io/guide/what-is-angular

Artieda Guachamin, C., Reza Morales, J., \& Rojas Cisneros, H. (2021). Sistema móvil para la oferta de recorridos con rutas fijas. Revista ODIGOS, 2(1), 21-40. https://doi.org/10.35290/ro.v2n1.2021.381

Jadhav, M. A., Sawant, B. R., \& Deshmukh, A. (2015). Single page application using angularjs. International Journal of Computer Science and Information Technologies, 6(3), 2876-2879.

Burbano Ulloa, E., Constante Molina, M., Hidalgo Guamán, L., \& Moya Chiluiza, F. (2020). Prototipo móvil para la geolocalización de mascotas callejeras. Revista ODIGOS, 1(3), 77-96. https://doi.org/10.35290/ ro.v1n3.2020.372

de la Maza Valles, A. (2020). API REST para la gestión de partidas multijugador de un juego serio. [Tesis de grado, Universidad de Valladolid]. UVaDOC http://uvadoc.uva.es/handle/10324/44417

Freeman, A. (2021). Understanding typescript. In Essential TypeScript 4 (pp. 35-41). Apress. https://doi. org/10.1007/978-1-4842-4979-6 2

González Díez, M. (2016). Clean architecture y RxJava en Android. [Tesis de grado, Universitat de Barcelona]. http://hdl.handle.net/2445/102790

Microsoft. (22 de enero de 2021). What is Azure DevOps? https://docs.microsoft.com/en-us/azure/devops/ user-guide/what-is-azure-devops?view=azure-devops

Microsoft. (s.f). Xamarin documentation. https://docs.microsoft.com/en-us/xamarin/

Paco Blanco, J., Fumero, A., Werterski, A., \& Rodríguez, P. (2009). Metodología de desarrollo ágil para sistemas móviles Introducción al desarrollo con Android y el iPhone. http://radar.oreilly.com/2008/07/interest-in-the-iPhone-android.html

Reyes Rivera, A. J., Vallejos Cango, M. de L., \& Quintana García, D. A. (2020). Nueva realidad: compras en línea y a domicilio. Revista ODIGOS, 1(3), 47-60. https://doi.org/10.35290/ro.v1n3.2020.370

Riera, E. G. (2020). Sistema de gestión documental para la empresa Logikard. Revista ODIGOS, 1(3), 61-76. https://doi.org/10.35290/ro.v1n3.2020.371

Solon Guimarães Jr, C., Bigolin Wiedemann, R., Rubio-Tamayo, L., \& Ventura Bayan Henriques, R. (2015). Propuesta de Front-end y Back-end para un Servicio Web Aplicado en Tecnología Asistiva.

Trasobares, A. H. (2003). Los sistemas de información: evolución y desarrollo. Proyecto social: Revista de relaciones laborales, (10), 149-165. 
Copyright (c) 2021 Juan Sebastián Reza Morales

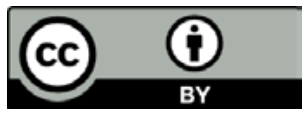

Este texto está protegido bajo una licencia internacional Creative Commons 4.0.

Usted es libre para Compartir - copiar y redistribuir el material en cualquier medio o formato - y Adaptar el documento - remezclar, transformar y crear a partir del material-para cualquier propósito, incluso para fines comerciales, siempre que cumpla las condiciones de Atribución. Usted debe dar crédito a la obra original de manera adecuada, proporcionar un enlace a la licencia, e indicar si se han realizado cambios. Puede hacerlo en cualquier forma razonable, pero no de forma tal que sugiera que tiene el apoyo del licenciante o lo recibe por el uso que hace de la obra.

$\underline{\text { Resumen de licencia - Texto completo de la licencia }}$ 